\title{
COVID-19-Associated Miller Fisher Syndrome: MRI Findings
}

\author{
(10.E. Lantos, ${ }^{-}$S.B. Strauss, and $\mathbb{1}^{\mathrm{E}} \mathrm{E}$. Lin
}

\begin{abstract}
SUMMARY: Miller Fisher syndrome, also known as Miller Fisher variant of Guillain-Barré syndrome, is an acute peripheral neuropathy that can develop after exposure to various viral, bacterial, and fungal pathogens. It is characterized by a triad of ophthalmoplegia, ataxia, and areflexia. Miller Fisher syndrome has recently been described in the clinical setting of the novel coronavirus disease 2019 (COVID-19) without accompanying imaging. In this case, we report the first presumptive case of COVID-19-associated Miller Fisher syndrome with MR imaging findings.
\end{abstract}

ABBREVIATIONS: $\mathrm{CN}=$ cranial nerve; COVID-19 = coronavirus disease 2019; MFS = Miller Fisher syndrome; CNS = central nervous system; PNS = peripheral nervous system

M iller Fisher syndrome (MFS), also known as Miller Fisher variant of Guillain-Barré syndrome, is an acute peripheral neuropathy that can develop after exposure to various viral, bacterial, and fungal pathogens. It is often immune-mediated and associated with anti-GQ1b antibodies, characterized by a triad of ophthalmoplegia, gait ataxia, and areflexia. Ophthalmoplegia is due to involvement of cranial nerves III, IV, or VI. Ataxia is thought to be due to cerebellar involvement, and areflexia is due to lower motor neuron involvement. MFS has recently been described in the clinical setting of the novel coronavirus disease 2019 (COVID-19) without accompanying imaging findings. ${ }^{1}$ While patients with COVID-19 typically present with fever, shortness of breath, and cough, neurologic manifestations, including headache, ataxia, cognitive impairment, anosmia, and stroke, have been reported. ${ }^{2-4}$ One retrospective review of 214 patients found neurologic symptoms in $36.4 \%$ of patients, with involvement of the CNS (24.8\%) greater than the peripheral nervous system $(8.9 \%) .{ }^{4}$ In this case, we report the first presumptive case of COVID-19-associated Miller Fisher syndrome with imaging.

A 36-year-old man with a remote history of left eye strabismus (asymptomatic for 30 years) was brought to the emergency department by ambulance, presenting with left eye drooping, blurry

Received April 8, 2020; accepted after revision May 2.

From the Department of Radiology, Weill Cornell Medical Center/New York Presbyterian Hospital, New York, New York.

Please address correspondence to Joshua E. Lantos, MD, Department of Radiology, Weill Cornell Medical Center/New York Presbyterian Hospital, 1305 York Ave, 3rd Floor, New York, NY 10021; e-mail: jol9057@med.cornell.edu

- Indicates open access to non-subscribers at www.ajnr.org

http://dx.doi.org/10.3174/ajnr.A6609

vision, and reduced sensation and paresthesia in both legs for 2 days. He was in his usual state of health until 4 days before presentation, when he developed viral symptoms in a COVID-19endemic region, reporting subjective fevers, chills, and myalgia. Physical examination was notable for a partial left third nerve palsy and decreased sensation below the knees to all modalities. MR imaging of the brain, including high-resolution imaging of the orbits and retro-orbital region, with and without gadolinium, was notable for striking enlargement, prominent enhancement with gadolinium, and T2 hyperintense signal of the left cranial nerve (CN) III (Figure). No other cranial nerves demonstrated abnormal signal or enhancement characteristics. MR imaging of the brain had normal findings. No cerebellar lesions were seen to explain the patient's ataxia. There were no findings of meningitis, encephalitis, demyelination, or infarction. MR imaging of the spine, which may have provided an imaging correlate for the patient's areflexia, was not performed.

The diagnosis of COVID-19 was confirmed by qualitative detection of Severe Acute Respiratory Syndrome coronavirus 2 RNA in a nasopharyngeal swab specimen by real-time reverse transcription polymerase chain reaction amplification and detection using TaqMan fluorescent oligonuecleotide probes (Altona Diagnostics) on the Rotor-Gene Q instrument. Serologic ganglioside antibody testing was performed by semi-quantitative enzymelinked immunosorbent assay (ARUP Laboratories) and showed Asialo GM1 antibody in the equivocal range, while testing for other antibodies, including anti-GQ1b, was negative. Nonetheless, the patient's hospital course was characterized by progressive ophthalmoparesis (including initial left CN III and eventual bilateral $\mathrm{CN}$ VI palsies), ataxia, and hyporeflexia, and the clinical picture 

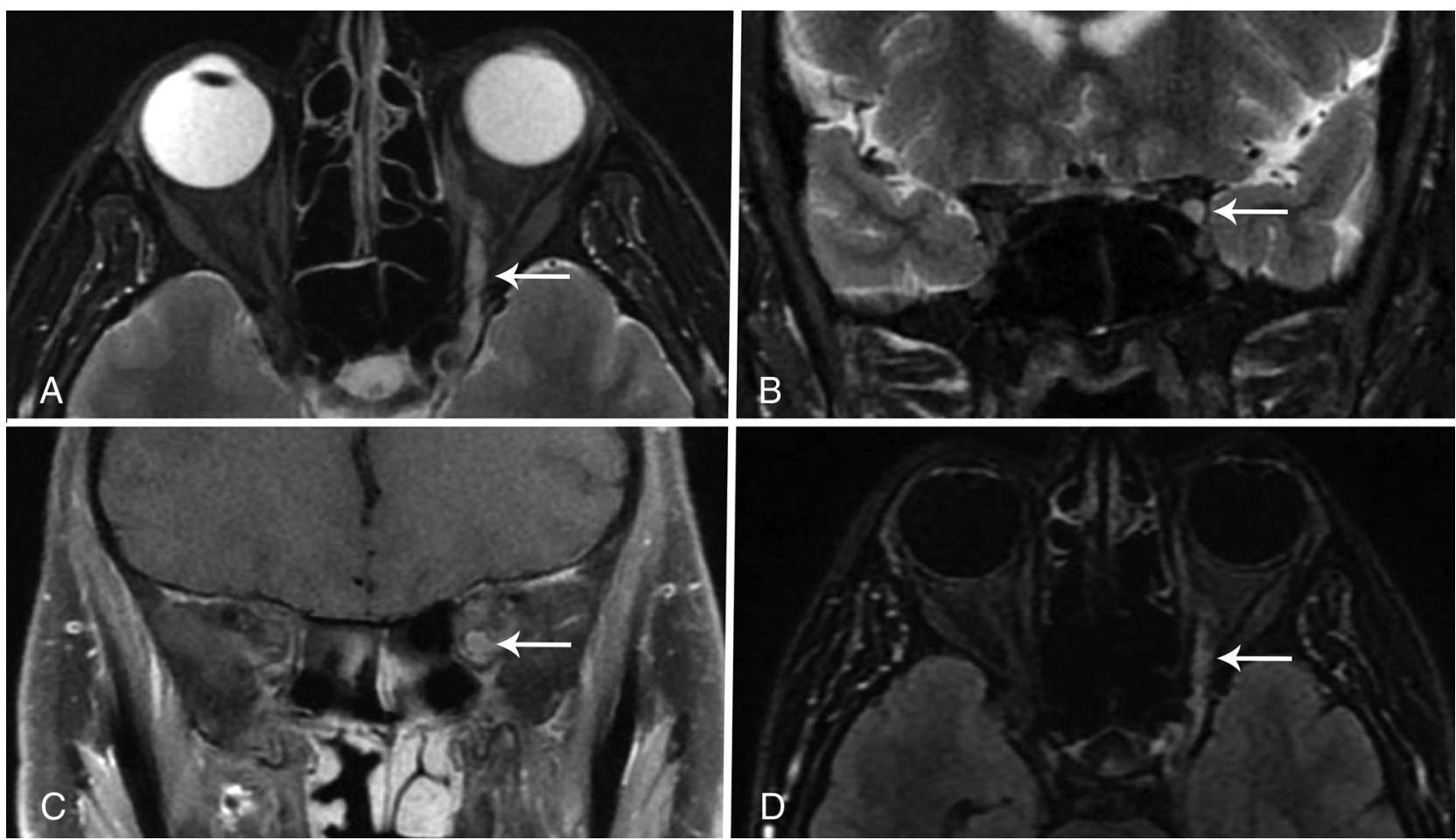

FIGURE. Axial and coronal T2-weighted fat-suppressed images through the orbits ( $A$ and $B$ ) demonstrate an enlarged CN III (arrow) with increased signal involving the nerve from the cavernous sinus through the orbit. Coronal Tl-weighted fat-suppressed postcontrast image $(C)$ also shows an enlarged CN III within the proximal orbit (arrow) and demonstrates marked enhancement of the nerve. The abnormal nerve is also visible on a whole-brain T2 FLAIR fat-suppressed postcontrast image (arrow, D).

was thought to be consistent with MFS from COVID-19 infection. The patient was treated with intravenous immunoglobulin, with subsequent improvement of neurologic symptoms. The patient also received hydroxychloroquine to treat the underlying COVID19 infection. No repeat MR imaging was performed, and the patient was discharged after 4 days of hospitalization.

MFS accounts for $1 \%-5 \%$ of cases of Guillain-Barré syndrome in Western countries, affects men twice as often as women, and is preceded by an upper respiratory illness in most patients. ${ }^{5}$ MFS presents commonly with diplopia (78\%), ataxia $(48 \%)$, or both $(34 \%){ }^{6}$ Our patient presented with symptoms of COVID-19 as well as diplopia, which was found to be due to a CN III palsy noted on the patient's clinical examination. MR imaging demonstrated corresponding enlargement, T2 hyperintensity, and enhancement of the affected CN III from the cavernous sinus through the orbit. This is the first presumptive case report of MFS associated with COVID-19 infection with imaging findings.

Although testing was negative for anti-GQ1b, the clinical picture was consistent with MFS, and the patient improved with treatment. Negative ganglioside antibody testing is a limitation of this report; however, a review of 123 patients with MFS found that $15 \%$ were negative for anti-GQ1b. ${ }^{7}$ The GQ1b ganglioside is a cell surface component that is concentrated in the paranodal regions, cranial nerves III, IV, and VI. Anti-GQ1b antibodies have been shown to bind to fractions of Campylobacter jejuni and Haemophilus influenzae and are thought to cause the symptoms of MFS through molecular mimicry. ${ }^{8}$
When antibody testing is negative in patients with MFS, symptoms may be due to viral neurotropism rather than immune-mediated injury. ${ }^{9}$ The functional receptor for COVID-19 is angiotensin-converting enzyme 2 and is present in neural tissue. ${ }^{4}$ Access to the CNS may be either hematogenous or via retrograde neural propagation along bipolar cells. Retrograde propagation along the olfactory pathway may account for the occurrence of anosmia in some patients with COVID-19. ${ }^{4}$ It has been proposed that retrograde propagation could lead to brain stem involvement and contribute to respiratory symptoms by affecting the nuclei that regulate respiratory rhythm such as chemoreceptors that detect changes in oxygen and $\mathrm{CO}_{2} .{ }^{10}$ Understanding of COVID-19 pathophysiology in the CNS and peripheral nervous system and its contribution to morbidity and mortality is still in its infancy. Whether MFS is the result of immune-mediated injury or viral neurotropism, this potential complication should be recognized by clinicians and radiologists so that appropriate treatment can be offered to these symptomatic patients.

\section{REFERENCES}

1. Gutierrez-Ortiz C, Mendez A, Rodrigo-Rey S, et al. Miller Fisher syndrome and polyneuritis cranialis in COVID-19. Neurology 2020 Apr 17. [Epub ahead of print] CrossRef Medline

2. Filatov A, Sharma P, Hindi F, et al. Neurological complications of coronavirus (COVID19): encephalopathy. Cureus 2020;12:e7352 CrossRef

3. Poyiadji N, Shahin G, Noujaim D, et al. COVID-19-associated acute hemorrhagic necrotizing encephalopathy: CT and MRI features. Radiology 2020 Mar 31. [Epub ahead of print] CrossRef Medline 
4. Mao L, Jin H, Wang M, et al. Neurologic manifestations of hospitalized patients with coronavirus disease 2019 in Wuhan, China. JAMA Neurol 2020 Apr 10. [Epub ahead of print] CrossRef Medline

5. Bukhari S, Taboada J. A case of Miller Fisher syndrome and literature review. Cureus 2017;9:e1048 CrossRef

6. Mori M, Kuwabara S, Fukutake T, et al. Clinical features and prognosis of Miller Fisher syndrome. Neurology 2001;56:1104-06 CrossRef Medline

7. Nishimoto $Y$, Odaka $M$, Hirata $K$, et al. Usefulness of antiGQ1b IgG antibody testing in Fisher syndrome compared with cerebrospinal fluid examination. J Neuroimmunol 2004;148:200-05 CrossRef Medline

8. Snyder LA, Rismondo V, Miller NR. The Fisher variant of GuillainBarre syndrome (Fisher syndrome). J Neuroophthalmol 2009;29:31224 CrossRef Medline

9. de Silva NL, Weeratunga P, Umapathi T, et al. Miller Fisher syndrome developing as a parainfectious manifestation of dengue fever: a case report and review of the literature. J Med Case Rep 2019;13:120 CrossRef Medline

10. Conde Cardona G, Quintana Pájaro LD, Quintero Marzola ID, et al. Neurotropism of SARS-CoV 2: mechanisms and manifestations. J Neurol Sci 2020;412:116824 CrossRef Medline 